PROCEEDINGS OF THE

AMERICAN MATHEMATICAL SOCIETY

Volume 139, Number 9, September 2011, Pages 3039-3043

S 0002-9939(2011)11018-9

Article electronically published on April 25, 2011

\title{
MONOMIALS AS SUMS OF POWERS: THE REAL BINARY CASE
}

\author{
MATS BOIJ, ENRICO CARLINI, AND ANTHONY V. GERAMITA
}

(Communicated by Irena Peeva)

\begin{abstract}
We generalize an example, due to Sylvester, and prove that any monomial of degree $d$ in $\mathbb{R}\left[x_{0}, x_{1}\right]$, which is not a power of a variable, cannot be written as a linear combination of fewer than $d$ powers of linear forms.
\end{abstract}

\section{INTRODUCTION}

It is well-known, and easy to prove, that if $k$ is a field of characteristic zero and $R=k\left[x_{0}, \ldots, x_{n}\right]=\bigoplus_{i=0}^{\infty} R_{i}$ is the standard graded polynomial algebra, then the $k$-vector space $R_{d}$ (for any $d$ ) has a basis consisting of polynomials $\left\{L_{1}^{d}, \ldots, L_{s}^{d}\right\}$, where $s=\left(\begin{array}{c}d+n \\ n\end{array}\right)=\operatorname{dim}_{k} R_{d}$ and the $L_{i}$ are pairwise linearly independent forms in $R_{1}$. It follows that every form in $R_{d}$ is a $k$-linear combination of at most $s d^{t h}$ powers of linear forms and, if $k$ is algebraically closed, simply a sum of at most $s$ $d^{\text {th }}$ powers of linear forms. We will call such a way of writing $F \in R_{d}$ a Waring expansion of $F$ because of the echo of Waring's problem from number theory. We will further refer to such an expression as a minimal Waring expansion for $F$ if the number of summands in such an expression for $F$ is minimal among all such representations.

If $n>0$ and $d=2$ it is a classical fact that although $s=\left(\begin{array}{c}n+2 \\ 2\end{array}\right)$, every quadratic form has a Waring expansion involving $\leq n+1<s$ summands. In general, i.e. for $[F]$ belonging to a non-empty Zariski open subset of $\mathbb{P}\left(R_{2}\right)$, a minimal Waring expansion for the quadric $F$ has exactly $n+1$ summands.

These observations have led to a series of problems, usually called Waring Problems, which ask for information on minimal Waring expansions for forms of degree $d$ in $R$.

The long outstanding problem of finding the number of summands in a minimal Waring expansion of the generic form of degree $d$ was solved, after being open for almost 100 years, by J. Alexander and A. Hirschowitz (see AH95]), when $k$ is an algebraically closed field of characteristic zero. (For positive characteristic, see [K99, Theorem 1.61].)

Of course, solving this problem for the generic form of degree $d$ does not always give information about any specific form of degree $d$, and the problem of finding the length of the minimal Waring expansion for specific forms has also been a continuing

Received by the editors May 17, 2010.

2010 Mathematics Subject Classification. Primary 14P99; Secondary 13A02, 15A21.

(C)2011 American Mathematical Society 
source of interesting speculations and lovely results. For example, it was Sylvester ([Har92]) who first observed that although for $R=\mathbb{C}\left[x_{0}, x_{1}\right]$, the generic form of degree $d$ has a Waring expansion with $s=\left\lceil\frac{d+1}{2}\right\rceil$ summands, the monomial $x_{0} x_{1}^{d-1}$ has $d$ summands in its minimal Waring expansion (the maximum possible).

The Waring problem for specific forms has been considered in depth by B. Reznick in his monograph (see Rez92]) and by G. Comas and M. Seiguer, who, to our knowledge, were the first to resolve the problem completely and algorithmically in $\mathbb{C}\left[x_{0}, x_{1}\right]$ (cf. [CS01]).

It is interesting to note that although the Waring problem is a very interesting and stimulating problem in purely algebraic terms, it has a surprising number of intimate connections with problems in areas as seemingly disparate as algebraic geometry and communication theory (see for example [RS00, CC03 and CM96).

Indeed, if $k=\mathbb{R}$, the field of real numbers, the connection with real world problems is very direct. This has prompted a reexamination of the Waring problem for $R=\mathbb{R}\left[x_{0}, x_{1}\right]$, and a recent, very suggestive paper of P. Comon and G. Ottaviani (see [CO09]) considered this very problem for degrees $d \leq 5$.

Our main result in this paper follows the line of Sylvester's examples and concerns the minimal Waring expansion for monomials in $\mathbb{R}\left[x_{0}, x_{1}\right]$. We first see how the Apolarity Lemma produces a simple proof of the fact that the minimal Waring expansion of the monomial $x_{0}^{a} x_{1}^{b}$ in $\mathbb{C}\left[x_{0}, x_{1}\right]$ with $0<a \leq b$ has $b+1$ summands. In sharp contrast to this we show that in $\mathbb{R}\left[x_{0}, x_{1}\right]$ every monomial of degree $d$ (except $x_{0}^{d}$ and $x_{1}^{d}$ ) has $d$ summands in its minimal Waring expansion.

\section{BASIC RESUltS}

Let $S=k\left[x_{0}, x_{1}\right]$ and $T=k\left[y_{0}, y_{1}\right]$, where $k$ is an algebraically closed field of chararcteristic zero. We make $S$ into a $T$-module using differentiation; i.e., we think of $y_{0}=\partial / \partial x_{0}$ and $y_{1}=\partial / \partial x_{1}$. We refer to a polynomial in $T$ as $\partial$ instead of using capital letters. In particular, for any form $F$ in $S_{d}$ we define the ideal $F^{\perp} \subseteq T$ as follows:

$$
F^{\perp}=\{\partial \in T: \partial F=0\} .
$$

The following Apolarity Lemma is a classical result whose proof can be found in [K99, Lemma 1.31].

Lemma 2.1. A homogeneous form $F \in S$ can be written as

$$
F\left(x_{0}, x_{1}\right)=\sum_{i=1}^{r} \alpha_{i}\left(L_{i}\right)^{d}, L_{i} \text { pairwise linearly independent, } \alpha_{i} \in k,
$$

i.e. has a Waring expansion with $r$ summands, if and only if the ideal $F^{\perp}$ contains the product of $r$ distinct linear forms.

\section{Binary monomials: The COMPlex Case}

The complex case is straightforward for monomials.

Proposition 3.1. Let $M=x_{0}^{a} x_{1}^{b}$ be a monomial in $\mathbb{C}\left[x_{0}, x_{1}\right]$. If $0<a \leq b$, then $M$ has a minimal Waring expansion with $b+1$ summands, i.e. is a sum of $b+1$ powers of linear forms and no fewer. 
Proof. Let $I=M^{\perp}=\left(y_{0}^{a+1}, y_{1}^{b+1}\right)$ and notice that the linear system defined by $I_{b+1}$ is base point free on $\mathbb{P}^{1}=\mathbb{P} S_{1}$. Applying Bertini's Theorem, we get that the generic element of $I_{b+1}$ defines a set of $b+1$ distinct points and hence it is the product of $b+1$ distinct linear forms. Thus the Apolarity Lemma yields that $M$ is the sum of $b+1$ powers of linear forms. If $r<b+1$, then $r$ powers do not suffice as no element in $I_{r}=\left(y_{0}^{a+1}\right)_{r}$ is a product of $r$ distinct linear forms.

\section{Binary monomials: The Real case}

We can also ask for a real Waring expansion of a monomial $M$. More precisely, we want to write

$$
M\left(x_{0}, x_{1}\right)=\sum_{i=1}^{r} \alpha_{i}\left(L_{i}\right)^{d}, \quad \alpha_{i} \in\{1,-1\},
$$

where the linear forms $L_{i}$ are in $\mathbb{R}\left[x_{0}, x_{1}\right]$. In order to do this, we have to increase the number of summands in Proposition 3.1 .

The following elementary facts will be extremely useful.

Lemma 4.1. Consider the degree d polynomial

$$
F(x)=c_{d} x^{d}+\cdots+c_{1} x+c_{0} \in \mathbb{R}[x] .
$$

If $c_{i}=c_{i-1}=0$ for some $1 \leq i \leq d$, then $F(x)$ does not have $d$ real distinct roots.

Proof. The proof is obvious if $i=1$ or $i=d$, so we may as well assume that $1<i<d$.

Consider all the pairs $\left(c_{r}, c_{s}\right)$ of non-zero coefficients such that $r>s$ and $c_{j}=0$ if $r>j>s$. Let $\alpha$ be the number of pairs such that $r-s$ is odd and $\beta$ the number of pairs such that $r-s$ is even. Notice that, by hypothesis, $\alpha+2 \beta<d-1$.

Now we apply Descartes' rule of signs. For a pair $\left(c_{r}, c_{s}\right)$ such that $r-s$ is odd we get a real root of $F(x)$. To see this notice that if $c_{r} c_{s}<0$, then we have a non-negative real root of $F(x)$. If $c_{r} c_{s}>0$, then we have a non-negative real root of $F(-x)$, hence a negative real root of $F(x)$. Similarly, for a pair $\left(c_{r}, c_{s}\right)$ such that $r-s$ is even we get either two real roots of $F(x)$ or none.

In conclusion, the number of real roots of $F(x)$ is at most $\alpha+2 \beta$, and we are done.

Lemma 4.2. For each $i<d$ there exists a degree $d$ polynomial $F(x)=c_{d} x^{d}+\cdots+$ $c_{1} x+c_{0} \in \mathbb{R}[x]$ having $d$ real distinct roots and such that $c_{i}=0$.

Proof. Choose $a_{1}, \ldots, a_{d} \in \mathbb{R}$ and consider the polynomial $F(x)=\left(x-a_{1}\right) \cdot \ldots$. $\left(x-a_{d}\right)$. This polynomial can also be written as

$$
F(x)=\sum_{i=0}^{d} E_{i}\left(a_{1}, \ldots, a_{d}\right) x^{i},
$$

where $E_{i}$ is the degree $i$ elementary symmetric function in its arguments. The vanishing of the $i$-th coefficient of $F(x)$ can be written as

$$
E_{i}\left(a_{1}, \ldots, a_{d-1}\right)+a_{d} E_{i-1}\left(a_{1}, \ldots, a_{d-1}\right)=0 .
$$

Hence, if we choose the $a_{1}, \ldots, a_{d-1}>0$ and distinct there exists a unique, negative value of $a_{d}$ such that the coefficient of $x^{i}$ in $F(x)$ is zero. As the roots of $F(x)$ are $a_{1}, \ldots, a_{d}$ the polynomial has $d$ real, distinct roots. 
Using the previous results we immediately get a lower bound on the number of summands in the minimal Waring expansion of a monomial in $\mathbb{R}\left[x_{0}, x_{1}\right]$.

Lemma 4.3. Let $M=x_{0}^{a} x_{1}^{b}$ be a monomial in $\mathbb{R}\left[x_{0}, x_{1}\right]$. If $0<a \leq b$, then $M$ does not have a Waring expansion with $r \leq a+b-1$ real summands.

Proof. Let $I=M^{\perp}=\left(y_{0}^{a+1}, y_{1}^{b+1}\right)$. The general degree $r$ element in $I$ has the form $F\left(y_{0}, y_{1}\right)$

$$
=c_{r} y_{0}^{r}+c_{r-1} y_{0}^{r-1} y_{1}+\cdots+c_{a+1} y_{0}^{a+1} y_{1}^{r-a-1}+c_{r-b-1} y_{0}^{r-b-1} y_{1}^{b+1}+\cdots+c_{0} y_{1}^{r} .
$$

If $a+1 \geq r-b+2$, then by Lemma 4.1. $F\left(y_{0}, y_{1}\right)$ is not the product of $r$ real linear forms. The conclusion follows by the Apolarity Lemma.

Proposition 4.4. Let $M=x_{0}^{a} x_{1}^{b}$ be a monomial in $\mathbb{R}\left[x_{0}, x_{1}\right]$. If $0<a \leq b$, then $M$ has a minimal Waring expansion with $a+b$ summands which are powers of real linear forms.

Proof. We have that $M^{\perp}=I=\left(y_{0}^{a+1}, y_{1}^{b+1}\right)$. Notice that $I_{a+b}$ is the subspace of $T_{a+b}$ of polynomials which are missing all the monomials having factor $y_{0}^{a}$ or $y_{1}^{b}$. Thus, Lemma 4.2 and the Apolarity Lemma yield the result.

Remark 4.5. A few months after this paper was submitted, Bruce Reznick posted an article on the arXiv Rez10 in which he considers the Waring expansion for binary forms over several fields $k$. Reznick's paper has many connections with this work, and, for example, his Corollary 4.11 contains our Propostion 4.4.

\section{ACKNOWLEDGEMENT}

This project started with a research visit supported by a grant for visiting researchers from the Göran Gustafsson Foundation.

\section{REFERENCES}

[AH95] J. Alexander and A. Hirschowitz. Polynomial interpolation in several variables. J. Algebraic Geom., 4(2):201-222, 1995. MR.1311347 (96f:14065)

[CC03] E. Carlini and J. Chipalkatti. On Waring's problem for several algebraic forms. Comment. Math. Helv., 78(3):494-517, 2003. MR1998391 (2005b:14097)

[CS01] G. Comas and M. Seiguer. On the rank of a binary form. Found. Comput. Math., 11 (1):65-78, 2010.

[CM96] P. Comon and B. Mourrain. Decomposition of quantics in sums of power of linear forms. Signal Processing, 53(2):93-107, 1996. Special issue on High-Order Statistics.

[CO09] P. Comon and G. Ottaviani. On the typical rank of real binary forms. arXiv:0909.4865v1, 2009.

[Har92] J. Harris. Algebraic geometry, A first course. Graduate Texts in Math., Springer-Verlag, New York, 1992. MR.1182558 (93j:14001)

[IK99] A. Iarrobino and V. Kanev. Power sums, Gorenstein algebras, and determinantal loci. Lecture Notes in Mathematics, 1721, Springer-Verlag, 1999. MR.1735271 (2001d:14056)

[RS00] K. Ranestad and F.-O. Schreyer. Varieties of sums of powers. J. Reine Angew. Math., 525:147-181, 2000. MR:1780430 (2001m:14009)

[Rez92] B. Reznick. Sums of even powers of real linear forms. Mem. Amer. Math. Soc., 96(463):viii+155, 1992. MR.1096187 (93h:11043)

[Rez10] B. Reznick. On the length of a binary form. Preprint arXiv:1007.5485. 
Department of Mathematics, KTH, SE-100 44 Stockholm, Sweden

E-mail address: boij@kth.se

Dipartimento di Matematica, Politecnico di Torino, 10129 Turin, Italy

E-mail address: enrico.carlini@polito.it

Department of Mathematics and Statistics, Queen's University, Kingston, Ontario, Canada, K7L 3N6 - And - Dipartimento di Matematica, Università di Genova, 16126 Genoa, Italy

E-mail address: Anthony.Geramita@gmail.com

E-mail address: geramita@dima.unige.it 